\title{
Germanica
}

\section{Le thème du retour d'exil dans la trilogie adenauérienne de Wolfgang Koeppen}

Die Rüekkehr des «Emigranten» in Koeppens Adenauerzeit-trilogie

\section{Pierre Vaydat}

\section{(2) OpenEdition}

1 Journals

Édition électronique

URL : http://journals.openedition.org/germanica/2810

DOI : 10.4000/germanica.2810

ISSN : 2107-0784

Éditeur

Université de Lille

\section{Édition imprimée}

Date de publication : 30 juin 1987

Pagination : 125-146

ISSN : 0984-2632

\section{Référence électronique}

Pierre Vaydat, "Le thème du retour d'exil dans la trilogie adenauérienne de Wolfgang Koeppen », Germanica [En ligne], 1 | 1987, mis en ligne le 28 septembre 2018, consulté le 06 octobre 2020. URL: http://journals.openedition.org/germanica/2810 ; DOI : https://doi.org/10.4000/germanica.2810

Ce document a été généré automatiquement le 6 octobre 2020.

(c) Tous droits réservés 


\title{
Le thème du retour d'exil dans la trilogie adenauérienne de Wolfgang Koeppen
}

Die Rüekkehr des «Emigranten» in Koeppens Adenauerzeit-trilogie

\author{
Pierre Vaydat
}

1 Voilà plus de trois décennies que les observateurs de la scène littéraire allemande s'interrogent sur un phénomène singulier : le « cas Koeppen », un tarissement brutal de la production romanesque chez un auteur jusque-là talentueux. En 1954 paraissait Der Tod in Rom, considéré aujourd'hui, par la force des choses, comme le dernier volet d'une trilogie décrivant la vie politique et sociale en Allemagne de l'Ouest au début de l'ère adenauérienne. Depuis, W. Koeppen semble avoir abandonné l'écriture fictionnelle, bien qu'il ait fait à plusieurs reprises quelques confidences, très réticentes à vrai dire, quant à une nouvelle œuvre en préparation. Dans l'entretien accordé en 1971 à Christian Linder ${ }^{1}$, Koeppen a éludé les questions relatives à un éventuel blocage dû à une déficience imaginative ${ }^{2}$. À cette date encore, il affirmait songer à un nouveau roman, mais celui-ci n'a jamais vu le jour.

2 Des critiques hostiles à Koeppen ont entrepris dernièrement de constater l'atrophie de ses facultés créatrices. C'est ainsi qu'un article acerbe du Spiegel conteste à notre auteur la qualité d'écrivain estimable et rappelle certains jugements négatifs formulés sur son art au moment même, déjà lointain, où ses romans lui valaient un début de renommée 3 . Presque simultanément ${ }^{4}$, Fritz J. Raddatz commente sans indulgence l'événement tout à fait mineur que représente à ses yeux la réédition de Die Mauer schwankt (paru en 1935). Après en avoir fustigé la prétendue nullité stylistique, Raddatz y relève, en s'appuyant sur des remarques de Karl Prümm ${ }^{5}$, certaines tonalités qu'il qualifie de fascisantes. Il conclut en se demandant, toujours après Karl Prümm, si l'acharnement mis par Koeppen après la guerre à dénoncer la persistance du nazisme et l'attitude pour le moins tolérante de la société ouest-allemande à l'égard de ceux qui s'étaient signalés par leur zèle au service du Troisième Reich n'était pas en quelque sorte une attitude compensatoire. Il semble que ni Koeppen, ni ses admirateurs, n'aient cru devoir 
répliquer à cette attaque. Engager une polémique contre l'article de Raddatz aurait d'ailleurs été inutile, puisque M. Reich-Ranicki avait montré, plus d'une dizaine d'années auparavant, que certains des passages du roman visé constituaient une critique de l'État policier, n'importe quel lecteur contemporain pouvant y reconnaître sans peine l'ambiance où il vivait lui-même ${ }^{6}$. Il s'agit en somme, pour les journalistes que nous venons d'évoquer, de laisser entendre que le succès tout relatif qu'avait connu Koeppen au début des années 50 était dû pour une trop large part au scandale d'un franc-parler passablement affecté.

3 À l'inverse, Ulrich Greiner, dans sa préface au volume collectif Über Wolfgang Koeppen ${ }^{7}$ avait tenté d'expliquer le mutisme du romancier en l'attribuant à l'incompréhension et à l'hostilité dont il avait été victime de la part des journalistes, du public et même des universitaires. Selon Greiner, l'échec de Koeppen romancier serait dû au fait qu'il a été le premier à publier en R.F.A. des romans authentiquement politiques, et cela au mauvais moment, bien trop tôt, c'est-à-dire à l'époque où la popularité du chancelier Adenauer était à son zénith. La véhémence de Koeppen aurait heurté les bien-pensants d'alors, ces suppôts d'une bourgeoisie redevenue triomphante; d'où leur réprobation, qui serait à l'origine des faibles tirages atteints par des romans auxquels les critiques de l'ère post-adenauérienne ne ménagent plus l'éloge. Les thuriféraires de la "restauration" auraient malmené Koeppen parce qu'ils avaient subodoré dans ses textes une menace pour une identité nationale hâtivement replâtrée, dont Koeppen démasquait les mensonges. Greiner, ce faisant, reprenait la thèse avancée par ReichRanicki quinze ans plus tôt ${ }^{8}$, et qui mettait en cause l'impact désastreux des comptes rendus. Mais on peut objecter à cet argument que les recensions étaient loin d'être unanimement défavorables. Du reste, l'hostilité d'une critique bien pensante a rarement suffi à frapper un écrivain de stérilité.

4 Il est donc peu vraisemblable que Koeppen ait cessé d'écrire des romans parce qu'il était accablé par cette déconvenue, d'autant plus qu'elle a été compensée par un indéniable succès d'estime : Koeppen recevait en 1962 le prix Büchner; en 1965, il se voyait décerner le prix de littérature de l'Académie des arts de Bavière; en 1967, il obtenait le prix Immermann de la ville de Düsseldorf ; et, la même année, le prix de la Fondation pour l'encouragement des écrivains à Munich. Si Koeppen n'est plus parvenu à faire œuvre de romancier, la cause de son silence n'est donc pas uniquement, ni même principalement, l'amertume d'un artiste ostracisé, doutant de ses moyens. Elle réside dans la conscience d'un isolement irrémédiable et inhibant, celui de l'écrivain retranché de sa nation. Pour Wolfgang Koeppen, le « retour d'exil » n'a pas fait renaître le sentiment d'une communion avec son peuple, bien au contraire.

5 Sous le Troisième Reich, Koeppen avait choisi, malgré l'option qui lui restait ouverte, de ne pas poursuivre une carrière dans le journalisme moyennant la soumission idéologique aux nouveaux maîtres de l'Allemagne. Il était allé vivre en Hollande. Ses deux premiers romans, publiés respectivement en 1934 et en 1935, Eine unglückliche Liebe et Die Mauer schwankt, l'avaient été chez Cassirer, éditeur suspect parce que juif. Ensuite, Koeppen était rentré en Allemagne, mais n'avait plus rien écrit, se contentant de laisser rediffuser Die Mauer schwankt par un nouvel éditeur, "aryen " celui-là, en 1939. En 1950, Koeppen reprend la plume. En l'espace de quatre années, il publie trois romans : Tauben im Gras (1951), Das Treibhaus (1953), et Der Tod in Rom (1954), soutenant donc un rythme de production plus qu'honorable. C'est alors que s'installe la paralysie. Lorsqu'on le questionne, l'écrivain refuse de l'expliquer, bien qu'il avoue en souffrir. 
6 Cette situation douloureuse du retour manqué après une longue période d'exil intérieur ou d'expatriation est le véritable thème constitutif de la trilogie adenauérienne. Elle détermine les comportements des personnages sympathiques, auxquels Koeppen a conféré des traits et des façons d'être qui sont les reflets de sa propre personnalité (ou du moins de ce que Koeppen, dont la discrétion est notoire en ce domaine, a consenti à en livrer). Philipp, Keetenheuve, les Kürenberg, Siegfried Pfaffrath, autant de figures pour qui l'Allemagne, même si elle a retrouvé sur le plan institutionnel le chemin de la démocratie, est devenue invivable. Pour ces êtres, l'exil est un état d'âme, ils sont devenus irrévocablement des étrangers dans leur pays d'origine. Ce thème insuffle à la trilogie son écrasante unité d'atmosphère ; l'impression d'accablement qui s'en dégage est un effet voulu par une poétique de l'aliénation dont Stanley Craven a vu en Koeppen un praticien exemplaire'. Quant aux personnages déplaisants, leur situation morale inverse nous fournit une contre-épreuve : ils demeurent des enracinés, évoluant avec aisance au sein d'un peuple pour lequel leurs antagonistes, ceux en qui Koeppen a figuré les justes, éprouvent désormais un éloignement générateur presque toujours de désespoir, débouchant sur l'impuissance à écrire et sur l'incapacité d'agir.

7 L'écrivain frappé d'improductivité que deviendra Koeppen lui-même, il l'a décrit comme par pressentiment dans son premier roman de l'après-guerre, Tauben im Gras. Philipp, certes, n'est pas Koeppen; il est à certains égards bien différent de son créateur, puisqu'il va par habitude parler de soi au docteur Behude, sans espérer d'ailleurs une aide quelconque de ce neurologue dont il est intellectuellement l'égal et dont il partage le nihilisme foncier. Cependant, Philipp est doté d'une biographie qui présente des analogies avec celle de Koeppen : il est l'auteur d'un roman interdit, puis oublié. Mais, à la différence de ce qui se produira plus tard pour Koeppen, Philipp est devenu incapable d'écrire ne fût-ce qu'une ligne, tant son environnement humain, celui du Munich de l'immédiat après-guerre, le rebute :

er hatte den Leuten, die draussen vorübergingen, nichts zu sagen. Die Leute waren verurteilt. Er war verurteilt. Er war in anderer Weise verurteilt als die vorübergehen den Leute. Aber er war auch verurteilt. Die Zeit hatte diesen Ort verurteilt. Si hatte ihn zu Lärm und Schweigen verurteilt. Wer redete, was redete man denn? WIE EMMY HERMANN GÖRING KENNENLERNTE, die grellen Plakate schrien es von allen Wänden. [...] Was sollte Philipp hier? Er war überflüssig ${ }^{10}$.

8 On peut évidemment interpréter la stérilité de Philipp en termes de situation existentielle, et non pas simplement comme le corollaire d'une situation historique. S. Craven s'y est employé, en évoquant le désarroi du sujet pensant face à un monde devenu inconnaissable, éclaté en fragments trop disparates pour que l'on puisse recomposer à partir d'eux cette totalité gratifiante au-delà des dissonances que devrait être une œuvre d'art ${ }^{11}$. Et il est exact que Koeppen a su transmettre l'image brisée d'un monde dépourvu de principe recteur et ordonnateur, d'où la "destination" de l'homme, chère autrefois à l'idéalisme allemand, s'est évaporée. Ce sentiment, Philipp en éprouve la hantise, et la vacuité existentielle qui le paralyse nous est communiquée par une phrase de son monologue intérieur où ce terme de "destination" est ironiquement ressassé :

Er fühlte sich wie eine der leeren Packungen, die der Besen zum Kehricht gefegt hatte, nutzlos, seiner Bestimmung beraubt. Welcher Bestimmung? War er zu etwas bestimmt gewesen, hatte er sich dieser Bestimmung entzogen, und konnte man sich überhaupt, vorausgesetzt es gab sie, einer Bestimmung entziehen ${ }^{12}$ 
Il reste que la désorientation et le vide dont souffre Philipp ne découlent pas de considérations philosophiques sur la relation nouvelle de l'homme à l'univers dépoétisé que lui présente la science moderne par la voix d'un autre personnage, le physicien Schnakenbach ${ }^{13}$, mais qu'ils sont bien la conséquence du nazisme :

[...] leider war Philipp kein bedeutender Sehriftsteller geworden, er war schliesslich nur jemand, der sich Sehriftsteller nannte, weil er in den Einwohnerakten als Schriftsteller geführt wurde; er war schwach, er war auf der Walstatt geblieben, auf der sich die sehändliche Politik und der gemeinste Krieg, Wahnsinn und Verbrechen ausgetobt hatten, und Philipps kleiner Ruf, der erste Versuch, sein erstes Buch war im Lautsprecherbrüllen und im Waffenlärm untergegangen, war von den Schreien der Mörder und der Gemordeten übertönt worden, und Philipp war wie gelähmt und seine Stimme war wie erstickt, und schon sah er mit Grauen wie der verfluchte Schauplatz, den er nicht verlassen konnte, vielleicht auch nicht verlassen mochte, für ein neues blutiges Drama hergerichtet wurde ${ }^{14}$.

10 Point de doute : l'écrivain se sent captif d'un pays qu'il exècre, mais qu'il ne peut quitter parce qu'il ne pourrait écrire nulle part ailleurs : et s'il abomine l'Allemagne, c'est pour des raisons qui sont mentionnées on ne peut plus explicitement. Ni la vision de l'univers en train d'exploser que rumine Schnakenbach, ni la conception d'une absurdité fondamentale de l'histoire qu'a faite sienne l'étudiante américaine Kay après l'avoir entendu exposer avec éloquence par son professeur, et que résume le vers de Gertrude Stein dont vient le titre du roman («Pigeons in the grass, alas») ne sont susceptibles d'abolir la souillure du récent passé. Philipp n'a pas milité dans les rangs hitlériens. Il ne se sent pas coupable. Mais il est enfermé dans une solitude qu'il ne peut surmonter, celle de la réconciliation impossible avec la patrie.

11 Le roman suivant, Das Treibhaus, est centré sur ce thème. Alors que Philipp n'était qu'une des multiples figures dont les destins s'entrecroisaient, ou parfois même se frôlaient sans s'influencer, Keetenheuve, député socialiste au Bundestag, est ici l'unique protagoniste, le seul personnage dont la vie intérieure est longuement restituée.

12 Cette fois, Koeppen a campé un émigré au sens propre, et non pas simplement un double de lui-même en situation d'exil intérieur :

Das war vor zwanzig Jahren gewesen, an dem Tag, an dem der Volkskommissar in das «Volksblatt» einzog. Die jüdischen Redakteure flogen gleich [...]; die anderen bekamen die Chance, sich $\mathrm{zu}$ bewähren. Keetenheuve verzichtete auf die Bewährung. Er holte sein Gehalt und reiste nach Paris. Er reiste freiwillig, und niemand hinderte ihn ${ }^{15}$.

13 Dès que le régime hitlérien s'est abîmé dans la défaite, Keetenheuve s'empresse de revenir, par patriotisme :

Er hatte nie aufgehört, sich als Deutscher zn fühlen ${ }^{16}$.

14 En idéaliste impénitent, il ambitionne de contribuer à édifier une nouvelle Allemagne démocratique. Il obtient sans difficulté un mandat de député S.P.D. Mais il s'aperçoit rapidement que tout converge vers la "restauration nationale » et se sent gagné par l'indignation morose, puis par le dégoût. Il en résulte pour lui une impuissance à agir qui est le pendant de l'impuissance à écrire dont souffrait Philipp dans le roman précédent. Ce qui n'était qu'esquissé dans le portrait de Philipp apparaît ici en pleine lumière : la situation oppressante de l'Allemand qui, après avoir fui le Troisième Reich, se retrouve, une fois rentré, isolé au milieu de compatriotes avec lesquels il n'a plus d'expérience historique commune, dont les réactions le navrent ou l'exaspèrent, et qu'envahit malgré lui une germanophobie obsessionnelle : 
Und wieder saß man in dem Käfig, in den man hineingeboren war, dem Käfig des Vaterlandes $[. . .]^{17}$

Keetenheuve de la nouvelle Allemagne. À travers les réminiscences et les réactions de Keetenheuve, Koeppen nous fait entrevoir la survie d'une Allemagne maléfique, dont l'apparition est bien antérieure aux causes les plus lointaines que l'histoire positive s'est efforcée d'assigner à la dictature hitlérienne :

[Keetenheuve] saß im Nibelungenexpress. Es dunstete nach neuern Anstrich, nach Renovation und Restauration; es reiste sich gut mit der deutschen Bundesbahn; und aussen waren die Wagen blutrot lackiert. Basel, Dortmund und die Schlote des Reviers ; Kurswagen Wien Passau, Fememörder Hagen hatte sich's bequem gemacht $[\ldots]^{19}$. au moment même où ses compatriotes s'épanouissent dans l'atmosphère du train de luxe, jouissant du confort et de la sécurité retrouvés. Le preux assassin du Nibelungenlied surgit en plein milieu d'une modernité qui s'ingénie à ne plus utiliser le mythe que sous la forme d'une appellation publicitaire. Presque exactement à l'époque où Koeppen écrivait son roman, Robert Minder donnait de la vieille épopée germanique un commentaire également imprégné du souvenir du fascisme, et dont le rapprochement avec la citation qui précède témoigne d'un regard identique sur l'Allemagne :

Dans cette rage concentrée, dans ce sado-masochisme gigantesque, quelle figure plus hitlérienne que le Hagen du Nibelungenlied [...] ? [...] par son mélange d'élan créateur et de violence destructrice, ne devait-il pas incarner aux yeux de la jeunesse allemande un certain existentialisme à la manière de Heidegger, le Heidegger du grand discours universitaire de 1933 qui ne laissait subsister au milieu de la ruine des valeurs traditionnelles que cette vertu suprême : « die Haltung », la «tenue intransigeante et soldatique »? [...] A [...] Siegfried déjà édulcoré et embourgeoisé, la génération des «Frontsoldaten», les Ernst Jünger, Wehner, Dwinger préféraient l'impitoyable, l'impénétrable Hagen ${ }^{20}$.

18 Cette présentation permet de saisir pourquoi Koeppen a choisi de réincarner l'archétypal personnage de Hagen dans les exécuteurs des corps francs et de l'« Organisation Consul», qui aimaient, on le sait, à se poser en continuateurs des justiciers de la Sainte-Vehme. Le terrorisme ponctuel des corps francs et des réseaux clandestins formés après leur dissolution a préparé l'avènement du nazisme, et la contiguïté idéologique des deux mouvements ne fait aucun doute ${ }^{21}$.

19 Avec Hagen devenu artisan et bénéficiaire du miracle économique, la rupture de l'année 1945, génératrice d'espérance en une mutation morale de la société allemande, se trouve oblitérée. La République fédérale, telle que la présente Koeppen, réintègre la continuité d'un devenir que façonne sans désemparer le vieil irrédentisme, en attendant les circonstances favorables à la résurrection d'un orgueil national destructeur. 
20 La « restauration », le retour aux structures de pouvoir du traditionnel État-puissance, n'est pas uniquement l'œuvre des partis conservateurs. Elle semble bien être aux yeux de Koeppen un phénomène ethno-psychologique : car le S.P.D., nous dit l'auteur, veut au fond lui aussi un relèvement national qui ne peut s'accomplir que si l'on pactise avec des strates déjà dominantes sous l'ancien ordre prussien et que l'on prend sur soi de faire toutes les concessions idéologiques impliquées par une telle option. Voilà ce que fait comprendre sarcastiquement au malheureux Keetenheuve le journaliste Mergentheim. Keetenheuve, moraliste fourvoyé dans la politique, avait cru possible d'édifier une Allemagne neuve, un modèle de pacifisme et de démocratie, en faisant appel à toutes les sensibilités qui s'étaient opposées à l'hitlérisme, fussent-elles conservatrices. Or, c'est l'opinion ouest-allemande dans son immense majorité qui approuve le rétablissement national conçu dans le vieil esprit militariste par les gouvernants de l'après-guerre. Si le soutien de l'électoral C.D.U./C.S.U., vers lequel ont reflué ceux qui votaient autrefois pour les nazis et les nationaux-allemands, va de façon parfaitement naturelle au chancelier, les électeurs S.P.O., insinue Koeppen, n'ont en dernière analyse guère plus de dispositions au progressisme. Le trait burlesque que contient la réplique de Mergentheim fait ressortir, par son outrance même, combien le S.P.O., après 1945, cherche à amadouer des masses demeurées chauvines en leur tréfonds, et dont il redoute les réactions viscérales :

Hier schau dir das Buch des Hohen Hauses an. Den Widerstand haben deine Kollegen schon wieder aus ihrem Lebenslauf gestrichen. Ich habe die neueste Auflage. Du scheinst noch bei der alten zu stehen. Und die ist schon eingestampft ${ }^{22}$.

Koeppen pousse ici son "vérisme poétique " jusqu'à la caricature, donnant vie à un possible latéral où les députés S.P.O., devenus des socialistes honteux, n'oseraient plus faire état de leur passé de résistants. On reconnait, à travers ce grossissement fantasmagorique, les appréhensions qui tourmentaient à l'époque les dirigeants du parti social-démocrate: ils craignaient que la présence dans leur sphère de trop nombreux émigrés ne suscite une réaction de rejet de la part des électeurs ${ }^{23}$.

Il faut se demander maintenant quel est au juste, dans la perspective de KoeppenKeetenheuve, l'ensemble des conditions politiques et sociales qui se trouve ainsi restauré. Pour notre auteur, la réponse est évidente, même si elle peut paraitre vague et criticable à un spécialiste des sciences politiques : le régime adenauérien, tel est en substance le diagnostic que Koeppen s'attache à nous communiquer, rétablit la domination d'une classe bourgeoise dont la mentalité et les méthodes n'ont pas évolué fondamentalement depuis le XIXe siècle. Le nazisme n'a été au fond que le prolongement de l'ère wilhelminienne, et la République fédérale prend le relais. En ce début des années 50, le patronat et les milieux d'affaires achèvent tout simplement de prendre leur revanche sur la révolution socialiste qui a échoué en 1919 et avorté durant les années 1946-1949. Telle est l'interprétation que dicte le rapprochement entre deux passages du roman. Le premier nous livre les réflexions de Keetenheuve assis à la terrasse d'un grand hôtel de Bad Godesberg avec vue sur le Rhin :

Jetzt mochten Manager sich hier erholen. Der Führer war eine Fehlinvestition gewesen, oder war er es nicht gewesen? Ein Dilettant sollte nicht urteilen. Vielleicht hatte sich der Retter rentiert. Wieviel Millionen Tote? Die Essen rauchen. Die Kohle wird gefördert. Die Erzöfen brennen. Weiß glüht der Stahl. [...] Tief in den Strom gesargt, im Bett neuer Sagen schwimmend, sagenhafte Bilanzen, die Volksmärchen der Abschreibungen, die Substanz unangetastet, [...] immer davongekommen $[. . .]^{24}$. 
Sorti indemne du crépuscule des dieux, le Capital a regagné les rives du Rhin. Le trésor des Nibelungen a échappé aux bombardements comme aux démontages. Il alimente la «serre chaude» du boom économique, dont l'ampleur et l'ambiance rappellent les lendemains rayonnants de 1871. Voilà les pensées qui viennent à l'esprit de Keetenheuve, alors que, pris de désœuvrement et d'angoisse, il déambule dans les rues de Bonn et s'est arrêté devant un magasin où l'on vend à nouveau des casquettes bigarrées et des rubans à porter en sautoir pour les membres des «Korporationen» estudiantines ultra-conservatrices :

Wirklich, die Gründerjahre waren zurückgekehrt, ihr Geschmack, ihre Komplexe, ihre Tabus ${ }^{25}$.

Si l'on pouvait disjoindre du reste du livre les attaques contre la "restauration ", il serait à la rigueur défendable de soutenir que Koeppen s'en prend à des forces politiques, à des structures sociales, mais non pas au peuple allemand en tant que tel. Or, il est impossible d'introduire dans l'analyse une séparation de cette sorte. Car, en même temps qu'il vitupère le retour à l'ordre moral ancien, Keetenheuve prend en aversion le peuple dont il a voulu être le représentant. Il s'interroge : qu'est-ce que le peuple, que veut-il exprimer ? $^{26}$ Les différentes réponses qui germent aussitôt dans sa réflexion toujours caractérisée par une dérive semi-onirique ne corroborent en aucune façon ses présupposés d'humaniste. Successivement, il évoque dans son monologue intérieur une jeunesse en uniforme galvanisée par les slogans hitlériens, des catégories que manipulent des groupes de pression, des individus sans cohésion mutuelle, atomisés par la diversité de leurs vouloirs pleins de bassesse. Même le cliché du peuple souffrant, qu'il ne manque pas de mobiliser, ne suffit plus à le convaincre qu'il croit encore à l'action bienfaisante du principe majoritaire. Dans son for intérieur, il est terrifié par l'Allemagne profonde, qu'il juge imprégnée d'antiparlementarisme et, bien pis, demeurée viscéralement hitlérienne, comme le prouvent les acquittements de criminels nazis par les jurés ${ }^{27}$. Plus on avance dans la lecture de Das Treibhaus, plus il apparaît que Keetenheuve déteste le peuple qu'il a entrepris de rédimer. Lorsqu'il va au cinéma, les actualités le perturbent. Pourtant, ce sont des scènes anodines qui défilent sur l'écran. Mais leur innocence même fait surgir le souvenir de l'hitlérisme, tant le banal, en Allemagne, reste proche du monstrueux pour qui ne s'est pas libéré du cauchemar de l'« époque brune »:

Der Präsident besuchte die Ausstellung. Ein Kind begrüsste ihn. Unser Führer liebt die Kinder. [...] Der Sport versöhnt die Völker. Zwanzigtausend starren auf einen Ball. Es ist höchst langweilig. Aber dann holt das Teleobjektiv der Filmkamera einzelne Gesichter aus den Zwanzigtausend heraus: erschreckende Gesichter, verkrampfte Kinnladen, hassverzerrte Münder, Mordgier im Blick. Wollt ihr den totalen Krieg? ja ja ja [...] War dies des Menschen Antlitz? War so erschreckend der Zeitgenossen Gesicht? ${ }^{28}$

Si Keetenheuve commence à haïr son peuple, c'est parce qu'il croit constater que celuici, personnifié une nouvelle fois par les petits cercles d'habitués des brasseries et des estaminets, éprouve à l'égard de la démocratie parlementaire, c'est-à-dire de la seule forme de régime qui soit véritablement conforme aux idéaux humanistes, une hostilité qui n'est pas simplement du scepticisme ou de l'aigreur, mais de la répulsion :

Er ging wieder in die weniger vornehme Weinstube. Die Stammtische waren besetzt. Die Stammtische erörterten die Abstimmung im Bundestag. Die Stammtische waren missgelaunt, und die Abstimmung missfiel ihnen. Aber ihr Missfallen und ihre Misslaune waren steril; sie waren eine Misslaune und ein Missfallen wie unter einem Vakuum. Die Stammtische nahmen übel. Auch jedes 
andere Ergebnis der Parlamentssitzung hätte sie missgelaunt gemacht und ihnen missfallen. Sie sprachen vom Bundestag mit einem prinzipiell vorhandenen Ärger; sie sprachen von der letzten Tagung wie von einem Ereignis, das zwar ärgerlich und von angemasster Macht sei, doch das sie nichts angehe und sie nicht berühre. Was berührte dieses Volk? Sehnten sie sich nach der Peitsche, um «Hurra» schreien zu können? ${ }^{29}$. Knurrewahn n'a plus jamais pu s'affranchir de l'esprit militaire. L'évolution intellectuelle et idéologique dont Koeppen a pourvu cette figure ${ }^{30}$ emprunte quelquesuns de ses traits à la personnalité de Kurt Schumacher, quelques autres à celle de Noske. Knurrewahn a beau se proclamer socialiste, matérialiste et athée, il demeure au point de vue psychologique un luthérien, attaché à des conceptions hiérarchiques, continuant d'accomplir ce qui ressemble à s'y méprendre à une mission terrestre. Il n'en prend conscience, au demeurant, que lorsque l'irritent les propos ou l'attitude de Keetenheuve, ce "vagabond» qui fait fi de l'autorité. Au temps de sa jeunesse, Knurrewahn avait cru à des valeurs : les lumières, le savoir, la justice sociale, facteurs de progrès. Le cours des événements lui ayant montré l'inanité de cet optimisme, il n'a plus désiré au fond que le pouvoir («Herrschaft und Macht»), tandis qu'à l'arrière-plan de son esprit subsistait la vague idée sinon d'améliorer le monde, du moins d'essayer de le conduire ailleurs qu'à la catastrophe :

Knurrewahn hatte viel durchgemacht; aber er war nicht weise geworden. Sein Herz war gut gewesen; nun hatte es sich verhärtet ${ }^{31}$.

31 L'embourgeoisement, l'esprit d'ordre conduisent Knurrewahn au conformisme patriotique. Grand blessé de la première guerre mondiale, il doit à la balle qui est restée dans sa poitrine, c'est-à-dire à l'exploitation d'un nationalisme latent dans l'opinion S.P.D., d'avoir été élu député au Reichstag. Victime de l'hitlérisme, il n'en demeure pas moins un partisan résolu de l'affirmation nationale. Koeppen s'est complu à imaginer 
un Kurt Schumacher démythifié, moins marqué par la souffrance, avec une bonne part d'étroitesse d'esprit en supplément, chez qui le calcul politique s'allie à la crédulité patriotique :

Knurrewahn [...] war ein nationaler Mann, und seine Opposition gegen die nationale Politik der Regierung war sozusagen deutschnational. Knurrewahn wollte der Befreier und Einiger des zerrissenen Vaterlandes werden, schon sah er sich als Bismarckdenkmal in den Knurrewahnanlagen stehen, und er vergass darüber den alten Traum, die Internationale. [...] Vielleicht fürchtete Knurrewahn mit Recht einen alten Fehler. Nach seiner Meinung war die Partei in der ersten deutschen Republik nicht national genug aufgetreten; sie hatte in der schon gespaltenen Internationale keinen Beistand gefunden, und in der Nation hatte sie die Massen verloren, die der eingängigen Parole des primitiven nationalen Egoismus folgten. Diesmal wollte sich Knurrewahn den nationalen Wind nicht aus den Segeln nehmen lassen. Er war für ein Heer, gebranntes Kind scheut nicht immer das Feuer, aber er war für eine Truppe von Patrioten [...], er war für Generale, aber sie sollten sozial und demokratisch sein ${ }^{32}$.

Il est caractéristique que les modèles d'identification de Knurrewahn soient sans exception des figures célèbres de l'histoire prussienne :

Er glich sich den Volksbiedermännern aus einer billigen patriotischen sozialen Traktätchenliteratur an, er wollte ein von Hysterie und Unmoral gereinigter Bismarck, ein Arndt, ein Stein, ein Hardenberg und ein klein wenig Bebel sein. Lassalle war ein Porträt des Abgeordneten als junger Mann [...] $]^{33}$.

Pour comprendre l'agencement de ce phantasme, il faut se souvenir que Bebel et Lassalle ont été élevés par Spengler à la dignité de "socialistes prussiens " ${ }^{34}$. En somme, Knurrewahn voudrait recommencer dans de meilleures conditions, en profitant de la disparition des couches sociales ultra-conservatrices ${ }^{35}$, l'histoire manquée de l'Allemagne au XIX ${ }^{e}$ siècle, en édifiant un État national de type fichtéen qui ne serait plus obéré par le féodalisme et réaliserait la fusion de l'ordre social parfait et de l'idéal humanitaire.

Cette démission des forces contestataires laisse le champ libre aux manœuvres du chancelier, mais aussi à la tendance technocratique incarnée par le personnage de Frost-Forestier. Dietrich Erlach suppose que cette figure a été inspirée par le général Gehlen, qui dirigeait à l'époque les services secrets ouestallemands ${ }^{36}$. M. Kaufmann émet de son côté l'hypothèse que "F.-F. pourrait désigner également Hans Globke ", l'homme de confiance d'Adenauer, ancien nazi et commentateur pointilleux des lois raciales édictées à Nuremberg, car il « exerce un pouvoir occulte au centre même du système politico-administratif, disposant notamment de postes de fonctionnaires à titre de prébendes $\|^{37}$. Ce qui semble en tout cas certain, c'est que Frost-Forestier, ancien officier supérieur de l'O.K.H. (Oberkommando des Heeres) symbolise le legs de l'esprit militaire prussien au nouvel État ouest-allemand. Il est le pendant négatif de Keetenheuve, en ce sens que ses actes s'inscrivent uniquement dans la rationalité de l'heure, sans référence à une norme éthique. Nulle trace, chez cet ancien militaire de haut grade, d'un remords ou simplement d'un sentiment de malaise à l'idée qu'il a servi les buts de conquête de l'hitlérisme avec autant de diligence et de méthode qu'il en apporte à seconder maintenant les gouvernants de Bonn :

Frost-Forestier erinnerte sich gern seiner Tätigkeit im O.K.H. Er liebte Soldatenausdrücke ${ }^{38}$. 
.

Dans le troisième roman, Der Tod in Rom, la germanophobie de Koeppen se fait plus âpre encore. À nouveau, le thème de l'exil, qu'il soit «intérieur» ou effectif, domine l'ensemble du livre.

À l'écrivain Philipp, à Keetenheuve, député, mais également traducteur de Baudelaire à ses heures, succède comme double de Koeppen un autre personnage d'artiste, le compositeur Siegfried Pfaffrath. Comme eux, il se sent frappé de malédiction par sa naissance :

[...] der Krieg war verloren, und er wenigstens war befreit worden und beugte sich nicht länger den Anschauungen der Sippe, in die geboren zu sein er immer nur entsetzlich gefunden hatte ${ }^{39}$.

Certes, le terme «Sippe», chargé de connotations ironiques, d'autant plus qu'il avait la faveur des nazis, ne désigne pas la collectivité allemande dans son ensemble, mais seulement la famille bourgeoise «deutschnational» dont Siegfried est issu et qu'il renie. Mais la suite du roman fait apparaître que la famille Pfaffrath est bien représentative des strates sociales dominantes dans une Allemagne où la «restauration » est désormais accomplie. Il est d'ailleurs remarquable que la gauche allemande, violemment persiflée dans Das Treibhaus, soit absente de ce dernier récit.

Du côté de Siegfried Pfaffrath, on trouve le couple Kürenberg: le chef d'orchestre, jouissant d'une renommée internationale, et son épouse juive se sont accommodés de l'exil. Ce trio de personnages est tout ce qui reste des aspirations humanitaires dans un monde déchu, et il est caractéristique que chez eux, non seulement le pouvoir créateur, mais également la fonction procréatrice, soient inhibés. Siegfried Pfaffrath n'aime guère sa propre musique, et lise Kürenberg y discerne les accents du désespoir, l'impuissance à écrire une œuvre achevée ${ }^{40}$. Les Kürenberg n'ont pas d'enfant. Pareillement, Siegfried Pfaffrath est horrifié à l'idée d'engendrer, et cette répugnance est l'un des facteurs qui le font basculer dans l'homosexualité ${ }^{41}$. Si les Kürenberg, contrairement à Siegfried, semblent connaître le bonheur, c'est qu'ils ont adopté un mode d'existence hédoniste, dédaignant les ancrages qui confèrent aux yeux des autres un sens à la vie :

Sie waren Exkursanten, die sich's in einer vielleicht unwirtlichen Welt wirtlich gemacht hatten und sich des Erdballs freuten. Kürenberg hatte sich auf Nomadie eingestellt ${ }^{42}$.

Pour l'exilé, il n'est pas de ré-enracinement possible, il peut, au mieux, aménager l'errance. C'est la leçon que tire des années de l'après-guerre Siegfried Pfaffrath :

[...] sich abf[in]den [...], ewig auf der Flucht zu sein, ewig auf einem Weg, von dem man wußte, daß er woher kam, aber nirgends wohin führte [...]. [...] es [...] schien mir [...] nicht mehr möglich zu sein, Deutschland zu ändern, man konnte nur sich ändern, und jeder mußte das für sich selbst tun, ganz allein [...]. ${ }^{43}$

En face des justes qui désespèrent ou cherchent à s'évader dans l'épicurisme se dressent les figures des notables qui continuent de régir le destin de l'Allemagne et qu'incarne la figure de Friedrich Wilhelm Pfaffrath, le père de Siegfried; il retrouve pendant ses vacances en Italie les gens de son bord, les immuables piliers de tous les régimes :

Friedrich Wilhelm Pfaffrath sass mit Anna, seiner Frau, und Dietrich, seinem jüngeren Sohn, im Gesellschaftsraum der von Deutschen bevorzugten Herberge, und schon hatten sie Anschluß an andere Italienreisende gefunden, Landsleute gleicher Schicht und gleicher Ansichten, Davongekommene, einmal vom Schreck Geschüttelte und dann Vergessende wie sie, Volkswagenbesitzer, Mercedesfahrer, 
an deutscher Tüchtigkeit Genesene und nun wieder willkommene Devisenbringer, sie unterhielten sich, tranken auch süssen Wermutwein, und auf den Tischen lagen Strassenkarten und Reisehandbücher, denn man beratschlagte Ausf]üge, wollte nach Tivoli und nach Frascati, aber auch ZUT wiederaufgebauten Abtei von Cassino, die Schlachtfelder waren zu besichtigen und waren diesen Menschen kein Grauen, und einer würde suchen und finden und rufen «hier stand unsere Batterie, hier spuckten wir "runter"», und dann würde sich's zeigen, was für ein feiner Kerl er war, mit Achtung, denn er bewunderte sich als fairen Krieger, als tötenden Sportsmann würde er vom Tommy sprechen und vom Ami und vielleicht sogar von den polnischen Legionären der Anders-Armee, aber das war nicht sicher, denn Pole blieb Pole $[. . .]^{44}$.

Les Pfaffrath, nationaux-allemands bon teint, sont ralliés pour la forme au régime de Bonn, bien qu'ils déplorent en privé les concessions qu'il a fallu faire à l'esprit de la démocratie occidentale. Mais ils demeurent indéfectiblement solidaires de l'ex-général S.S. Judejahn, beau-frère de Friedrich Wilhelm, un criminel de guerre réfugié dans un pays arabe dont il entraîne les troupes et qui est venu à Rome pour y négocier des achats d'armement.

Judejahn connaît l'exil de fait mais l'ignore avec superbe comme condition morale. Il compte bien revenir s'intégrer à une Allemagne à laquelle il s'identifie sans façon et qui continue tout naturellement à le reconnaître pour l'un des siens :

Wo Judejahn befahl, war Preussens alte Gloria, und wo Judejahn hinkam, war sein Grossdeutsehland. Der Sand der Wüste war noch immer der Sand der Mark. Judejahn war verjagt, aber er war nicht entwurzelt $[. . .]^{45}$

À Rome, Judejahn reprend contact sans difficulté avec sa famille et avec d'autres touristes allemands, il s'offre même le luxe de se sentir moralement supérieur aux bourgeois qui ont trahi le Führer et se sont ré-enrichis sans vergogne après l'effondrement du Troisième Reich. Et s'il est vrai que son épouse Éva, qu'il a délaissée, se sent en exil dans le monde nouveau de la R.F.A., dont la population s'est distanciée trop facilement à son gré de la foi nazie, elle n'en promet pas moins à l'ex-général S.S., lorsque celui-ci accepte finalement de la rencontrer, que ses compatriotes, en dépit du changement de régime, l'accueilleront avec respect et affection :

Du brauehst keinen falsehen Namen, du brauchst keine Brille, du benötigst keinen Pass. Die Grenzer werden sagen «sind Herr General wieder da? wir freuen uns, dan Herr General wieder hier sind», und sie werden vor dir strammstehen und werden dich reisen lassen, wohin du willst, und sie werden stolz sein, mit dir gesprochen zu haben, und zu Hause wird man dich mit Böllerschüssen empfangen und du wirst unantastbar sein ${ }^{46}$.

Rassuré sur ce point, Judejahn fait part aux Pfaffrath de son intention de revenir au pays :

Judejahn wollte nach der SouveräniHitsverleihung ${ }^{47}$ in Deutschland erseheinen, und Pfaffrath niekte, dann habe es keine Gefahr mehr, keine deutsehe Behörde würde ein Nürnberger Urteil vollstrecken und kein deutsehes Gericht würde Judejahn verdammen $[. . .]^{48}$.

Il ne s'agit pas ici de juger la valeur analytique de ce tableau, auquel on a reproché non sans précipitation d'être une caricature. Mais il est aisé de comprendre, après avoir lu la trilogie adenauérienne, pourquoi Wolfang Koeppen a renoncé, sans trouver un moment vraiment opportun pour le proclamer expressément, à la production romanesque. L'exil intérieur indéfiniment prolongé a vraisemblablement fait naître en lui un sentiment d'éloignement insurmontable à l'égard de son propre peuple. Or, le roman, et surtout le roman d'actualité, exige de l'artiste l'immersion dans la vie 
nationale et par conséquent un minimum de sympathie même au plus fort de la confrontation. Aussi était-il logique que W. Koeppen se cantonnât désormais dans la critique littéraire, le fragment autobiographique ou la relation de voyage.

\section{NOTES}

1. Über Wolfgang Koeppen, herausgegeben von Ulrich Greiner, édition Suhrkamp 864, 1976, pp. 257-276. Voir également, du même Ch. Linder, «Im Übergang zum Untergang. Zum Schweigen Wolfgang Koeppens», Akzente, $\mathrm{n}^{\circ} 19,1972$, p. 41-63, qui est un commentaire de cet entretien.

2. Voir Über Wolfgang Koeppen, ibid., notamment p. 262.

3. «Der eiserne Griff der Verehrer. Spiegel-Redakteur Christian Schluze-Gerstein über den Koeppen-Kult», Der Spiegel, 24.01.1983.

4. «Neuer Blick auf Koeppen. Zu seinem frühen Roman Die Mauer schwank», «Die Zeit», 11.03.1983.

5. Paru dans Schreibheft, revue que nous n'avons pu consulter.

6. Voir M. Reich-Ranicki, Deutsche Literatur in West und Ost, Rowohlt, Hamburg, 1970, p. 29-30.

7. Über Wolfgang Koeppen, p. 12.

8. Voir «Der Fall Wolfgang Koeppen. Ein Lehrbeispiel dafür, wie man in Deutschland mit Talenten umgeht», Die Zeit, 8.9.1961, réimprimé dans Über Wolfgang Koeppen, p. 101-118.

9. Voir Stanley Craven, Wolfgang Koeppen, A Study in Modernist Alienation, Akademischer Verlag Hans Dieter Heinz, Stuttgart, 1982

10. Tauben im Gras, Suhrkamp Taschenbuch 601, 1980, p. 53.

11. S. Graven, idem, p. 185.

12. Tauben im Gras, p. 25.

13. Ibid., p. 106.

14. Ibid., p. 96.

15. Dos Treibhaus, Suhrkamp Taschenbuch 78, 1981, p. 32.

16. Ibid., p. 12.

17. Ibid., p. 68-69.

18. Ibid., p. 85.

19. Ibid., p. 7.

20. Robert Minder, Allemagne et Allemands, Éditions du Seuil, Paris, 1949, pp. 249-250.

21. Sur la filiation entre l'esprit des corps francs et la mentalité des S.S., voir Hans Buchheim, «Befehl und Gehorsam», dans Anatomie des S.S.-Slaates, dtv 462, 1967, t. 1, p. 216 sq. Sur l'histoire des corps francs, consulter notamment Robert G. Waite, Vanguard of Nazism. The Free Corps Movement in Postwar Germany, 1918-1923, Harvard University Press, 1952; on trouvera dans l'appendice de cet ouvrage une liste de membres des corps francs ayant ensuite assumé des fonctions de commandement dans la S.A. Point de vue opposé chez Hannsjoachim Koch, Der deutsche Bürgerkrieg. Eine Geschichte der deutschen und österreichischen Freikorps, 1918-1923, Ullstein, 1978, ouvrage écrit dans un esprit de révision nationaliste.

22. Das Treibhaus, p. 67.

23. Sur ce sentiment d'infériorité très répandu dans le S.P.D. de l'époque vis-à-vis de la «méfiance populaire», voir A. Grosser, L'Allemagne de notre temps, éd. Fayard-Livre de Poche, collection « Pluriel », 1978, p. 282.

24. Das Treibhaus, p. 92. 
25. Ibid., p. 120.

26. Ibid., p. 35.

27. Ibid., p. 36.

28. Ibid., p. 123.

29. Ibid., p. 181.

30. Ibid., p. 67 et p. 75 sq.

31. Ibid., p. 76.

32. Ibid., p. 77-78.

33. Ibid., p. 78.

34. Voir Oswald Spengler, Preußentum und Sozialismus, 1919, dans Politische Schriften, С.H. Beck'sche Verlagsbuchhandlung, München und Berlin, 1934, p. 10 : «In der Bebelpartei war etwas Soldatisches gewesen, das sie vor dem Sozialismus aller anderen Länder auszeichnete; klirrender Schritt der Arbeiterbataillone, ruhige Entschlossenheit, der Mut, für etwas Jenseitiges zu sterben». Et, ibid., p. 69 : «Lassalle war es, der 1862 in seiner Schrift Was nun? die Verbindung des preußischen Königtums mit der Arbeiterschaft zum Kampfe gegen den Liberalismus und die englische "Nachtwächtertheorie" des schwachen Staates verlangt hat.»

35. Selon une opinion très répandue chez les sociologues de la R.F.A., le brassage des strates sociales opéré par le régime hitlérien, puis prolongé par la défaite, aurait démantelé la structure très hiérarchisée de la société wilhelminienne, encore bien vivante sous Weimar. Telle est la thèse soutenue notamment par Golo Mann ; voir sa Deutsche Geschichte im 19. und 20. Jahrhundert, S. Fischer Verlag, éd. 1966, p. 1027: «Man kann einen Thron restaurieren, nicht eine Gesellschaftsordllung. Der deutsche Obrigkeitsstaat, dessen herrschende Schichten Beamtentum, Justiz, Kirche, Heer, Adel - den Sturz der Hohenzollern überlebten, und der die Weimarer Republik ruinieren half, ist in den Jahren Adolf Hitlers zugrunde gegangen; den Jahren einer sehr stark aus dem Untersten kommenden, die Klassen durcheinander wirbelnden, wurzellosen und entwurzelnden, vulgären und totalen Diktatur, die mit der alten Autorität nichts, mit der alten Hierarchie wenig mehr zu tun hatte. Der stärkste Träger der alten Autorität, der preußische Adel, ist dreimal ruiniert worden: Durch den Krieg, in dem Tausende seiner Mitglieder fielen; durch seinen Widerstand gegen Hitler, die Kette von Verschwörungen, in deren Folge er zu Hunderten ausgerottet wurde, endlich durch Polen und Russen [...]».

36. Voir Dietrich Erlach, Wolfgang Koeppen als zeitkritischer Erzähler, Acta Universitatis Upsaliensae, Upsala, 1973, p. 145.

37. M. Kauffmann, «Das Treibhaus - un roman à clés», Cahiers de l'Institut d'allemand d'Asnières, Université de Paris III, 1982, p. 94.

38. Das Treibhaus, p. 31.

39. Der Tad in Rom, Suhrkamp Taschenbuch 241, p. 10.

40. Ibid., p. 78.

41. Ibid., p. 160.

42. Ibid., p. 44.

43. Ibid., p. 112-113.

44. Ibid., p. 30-31.

45. Ibid., p. 24.

46. Ibid., p. 141.

47. Rappelons que les accords de Bonn ont conféré la souveraineté à la R.F.A. le 26 mai 1952.

48. Ibid., p. 158. 


\section{RÉSUMÉS}

Depuis 1945, Wolfgang Koeppen, romancier d'un certain renom à l'époque adenauérienne, n'a plus fait paraître d'œuvre fictionnelle et ce mutisme a suscité la stupéfaction du public. Les critiques littéraires continuent de chercher des raisons à ce tarissement de sa production, et de récents articles de presse ont émis le soupçon que Koeppen aurait été surestimé durant les années 50 et qu'il aurait perdu depuis longtemps sa puissance créatrice. Les défenseurs de Koeppen font valoir de leur côté que des campagnes haineuses contre ses romans politiques, jugés mal venus en pleine époque de restauration ", seraient responsables du blocage. On a tenté dans le présent article d'interpréter d'une manière nouvelle le silence de Koeppen en se fondant sur l'analyse de la thématique du retour manqué dans Tauben im Gras, Das Triebhaus et Der Tod in Rom: la véritable origine du blocage résiderait non dans un fléchissement de la puissance créatrice mais dans le sentiment d'éloignement de Koeppen vis-à-vis de son propre peuple. Même après 1945, Koeppen n'a pu surmonter l'exil intérieur : les scènes de la vie nationale en R.F.A. au temps du miracle économique, de l'intégration dans le système politique occidental et du réarmement illustrent cet éloignement et font apparaître que, pour Koeppen, derrière un démocratisme de fraîche date, les anciennes forces maléfiques sont toujours à l'œuvre dans la psyché du citoyen de la R.F.A., si bien que le juste est isolé et devient forcément de moins en moins capable d'établir des contacts humains, d'écrire et d'agir, un destin que Koeppen a encore su décrire avant d'y succomber.

Seit 1954 hat Wolfgang Koeppen, ein namhafter Romanautor der Adenauerieit, kein fiktionales Werk mehr veröffentlicht, ein Verstummen, das vom Publikum mit Befremden wahrgenommen wurde. Literaturkritiker suehen immer noch nach Gründen für dieses Versiegen seiner Produktion, wobei in jüngst ersehienenen Zeitungsartikeln der Verdacht auftauchte, Koeppen sei in den 50er Jahren überschatzt worden und habe seine schöpferische Kraft längst eingebüßt. Koeppens Verteidiger behaupten ihrerseits, gehässige Kampagnen gegen seine in der Restaurations periode unwillkommenen politischen Romane trügen die Schuld an seiner Schreiblähmung. Es wird hier versucht, Koeppens Schweigen anhand der Thematik der verfehlten Heimkehr in Tauben im Gras, Das Treibhaus, und Der Tod in Rom neu zu deuten : als eine Hemmung, die nicht in nachlassender Schöpferkraft, sondern in der Entfremdung vom eigenen Volk ihre wahre Wurzel hat. Die Situation des inneren Exils konnte Koeppen auch nach 1945 nicht überwinden: die Bilder aus dem bundesdeutschen Volksleben in der Zeit des Wirtschaftswunders, der Westintegration und der Wiederbewaffnung lassen erkennen, daß für Koeppen hinter dem frischen demokratischen Anstrich die alten bösen Kräfte unbeirrt weiterwirken in der Psyche des Bundesbürgers, so daß der vereinsamte Gerechte zunehmend kontaktarm, schreib- und handlungsunfähig werden muß, ein Schicksal, das Koeppen noch zu schildern verstand, bevor er ihm selber erlag.

\section{AUTEUR}

PIERRE VAYDAT

Université de Lille III 